\title{
Tecnologías de la información y las comunicaciones en Colombia: política de telecomunicaciones sociales Compartel: un caso de estudio
}

\section{Information and Communication Technologies in Colombia: Social Telecommunication Policy - Compartel: A Case Study}

\section{Magally Hernández Ospina (Colombia)}

Universidad Santo Tomás magallyhernandez@usantotomas.edu.co

\section{Resumen}

Este texto presenta la caracterización, inicio, implementación y desarrollo de la política pública en telecomunicaciones sociales Compartel llevada a cabo en Colombia entre 1998-2009 a partir de una contextualización de lo que son las políiticas públicas. Destaca los principales momentos de cambio de Compartel, los actores que intervinieron
Abstract
This paper presents the characterization, initiation, implementation and development of social policy in telecommunications, Compartel, which was conducted in Colombia from 1998 to 2009 , based on a contextualization from public policies. It also highlights Compartel's key moments of change, its actors, characteristics, and results

$\begin{array}{ll}\text { FECHA DE RECEPCIÓN: } & 26 \text { de agosto de } 2011 \\ \text { FECHA DE REVISIÓN: } & 16 \text { de febrero de } 2012 \\ \text { APROBACIÓN: } & 21 \text { de marzo de } 2012\end{array}$

FECHA DE RECEPCIÓN:

FECHA DE REVISIÓN:

16 de febrero de 2012

Para citar Este artículo / to cite this ARTicle

Hernández Ospina, M. Tecnologías de la información

y las comunicaciones en Colombia: política de

telecomunicaciones sociales Compartel: un caso de estudio. Poliantea 8 (14), 11 3-144. 
en ellos, las características y resultados propiciados por los cambios de política, y ofrece algunas conclusiones sobre la evolución de las políticas públicas de las tecnologías de la información y las comunicaciones (TIC) en Colombia, relacionadas directamente con la política de telecomunicaciones sociales Compartel. Esta ponencia surge a partir de la investigación realizada por la autora al cursar la maestría en Estudios Políticos en el Instituto de Estudios Políticos y Relaciones Internacionales (lepri), de la Universidad Nacional de Colombia. La metodología utilizada para el desarrollo de la investigación fue de tipo cualitativa, con la utilización de técnicas de investigación como entrevistas semiestructuradas y análisis documental de un compendio de documentos en un periodo de once años, específicamente, entre 1998 y 2009. Para el desarrollo de esta investigación se aplicó el modelo de análisis de política pública denominado Advocacy Coalitions.

Palabras clave: políticas públicas, Advocacy Coalitions, tecnologías de la información y las comunicaciones, Internet, Compartel. coming from policy changes. In addition, this paper presents conclusions on the evolution of ICT policies in Colombia, directly related to the social telecommunication policy, Compartel. The author researched about this topic when studying a Master's degree in Political Studies at Instituto de Estudios Políticos y Relaciones Internacionales, IEPRI, of Universidad Nacional de Colombia. The methodology used for the development of the research was qualitative, by using research techniques such as semi-structured interviews and documentary analysis of a compendium of documents in a period of eleven years, specifically between 1998 and 2009. For the development of this research, the model of public policy analysis called Advocacy Coalitions was applied.

Keywords: Public policies, Information and Communication Technologies, Internet, Compartel. 


\title{
Tecnologías de la información y las comunicaciones en Colombia: política de telecomunicaciones sociales Compartel: un caso de estudio
}

\begin{abstract}
Magally Hernández Ospina (Colombia)
Comunicadora Social-Periodista, especialista en Comunicación Organizacional por la Universidad Autónoma de Occidente, Cali; magíster en Estudios Políiticos por el Instituto de Estudios Políiticos y Relaciones Internacionales (lepri), de la Universidad Nacional de Colombia, sede Bogotá; diplomada en Didáctica, Pedagogía y Evaluación, así como en Televidencia Crítica. Actualmente se desempeña como docente investigadora en la Facultad de Comunicación Social para la Paz, donde también coordina el Comité Curricular y de Perfeccionamiento Docente y el Comité de Evaluación y Autorregulación.
\end{abstract}

\section{Introducción}

En las últimas tres décadas, uno de los intereses del Gobierno colombiano ha sido dinamizar el sector de las TIC en el país, interés que se ve reflejado en los lineamientos de política enunciados en los planes de desarrollo de las administraciones de Andrés Pastrana Arango, Álvaro Uribe Vélez y Juan Manuel Santos Calderón.

Este interés ha sido caracterizado por considerar las TIC como herramientas claves para el desarrollo económico del país, tecnologías a las que define la Ley 1341 de 2009 como "el conjunto de recursos, herramientas, equipos, programas informáticos, aplicaciones, redes y medios que permiten la compilación, procesamiento, almacenamiento, transmisión de información como: voz, datos, textos, video e imágenes". Especialmente, la mirada se ha centrado en que estas tecnologías permitan, en primera instancia: desarrollar en el país una economía basada en el conocimiento (factor clave para el desarrollo), al facilitar su adquisición, absorción y comunicación, y en segundo lugar, el uso masivo en el país de las TIC permite crear un entorno económico 
atractivo, a la vez que facilita la participación de la sociedad colombiana en la nueva E-conomía, esta última, basada "en la interacción entre las tecnologías de información y la economía tradicional" (Consejo Nacional de Política Económica y Social [Conpes] 3072; Departamento Nacional de Planeación [DNP], 2000, pp. 5-6).

Todo esto en el marco de unos objetivos gubernamentales que propenden por la construcción y consolidación de un Estado más moderno que brinde a sus ciudadanos la oportunidad de acceder a servicios y trámites por medio de la red Internet y la garantía de universalizar el acceso a la información y al conocimiento como elementos claves para el desarrollo económico.

De acuerdo con lo anterior, esta ponencia esboza los antecedentes de la política de telecomunicaciones sociales Compartel y su implementación y resultados en Colombia entre 1998 y 2009 como una de las políticas públicas más representativas en el país en materia de TIC, particularmente telefonía social e Internet en sentido social. Para esto se presentará, en una primera parte, una contextualización a partir de la definición teórica de las políticas públicas, sus modelos de análisis y el modelo utilizado en esta investigación, denominado Advocacy Coalitions, propuesto por Paul Sabatier. En un segundo momento se enunciará la metodología de tipo cualitativo utilizada; en la tercera parte se describirá la política de telecomunicaciones sociales Compartel, se identificarán algunos elementos claves del modelo Advocacy Coalitions y, por último, se presentarán algunas de las conclusiones de la investigación realizada.

\section{Contextualización de Compartel a partir de las políticas públicas}

En español el término política es definido por la Real Academia Española mediante doce acepciones, entre las que se encuentran lo perteneciente o relativo a la doctrina política; lo perteneciente o relativo a la actividad política, arte, doctrina u opinión referente al gobierno de los Estados; actividad de quienes rigen o aspiran a regir los asuntos públicos, así como la actividad del ciudadano cuando interviene en los asuntos públicos con su opinión, su voto o de cualquier otro modo (www. rae.es). En el idioma inglés el término política tiene tres formas etimológicas: policy, politics y polity, las cuales evitan la confusión de sus significados; por lo tanto, al referirnos a las políticas públicas se hace necesario remitirse a 
las acepciones inglesas. Así, el término policy denota "lo que el Gobierno opta por hacer o no hacer" (Dye, citado en Vargas, 1999, p. 56); politics, definida como la actividad política, y polity, referida al Gobierno (Roth, 2006); en este sentido las políticas públicas se relacionan con la forma etimológica policy, la cual, de acuerdo con Salazar (1999, p. 50), es "el conjunto de sucesivas respuestas del Estado (o de un Gobierno específico) frente a situaciones consideradas socialmente como problemáticas", en este sentido las políticas públicas son todas aquellas acciones de los entes gubernamentales y sus funcionarios, que propenden por la solución o no solución de diversas situaciones de necesidad o riesgo para los ciudadanos de una nación.

A su vez, se debe tener en cuenta que toda política pública está constituida por etapas tales como la identificación del problema y ordenación de la agenda; la formulación, adopción, implementación y evaluación de la política, tal y como lo señala James Anderson (citado en Nelson, 2001, p. 802). Así mismo, diversos autores señalan que el proceso de política

Tabla 1. Ciclo de la política pública.

\begin{tabular}{|c|c|c|c|c|}
\hline Fase I & Fase II & Fase III & Fase IV & Fase V \\
\hline $\begin{array}{l}\text { Identificación e } \\
\text { institucionalización de un } \\
\text { problema }\end{array}$ & $\begin{array}{l}\text { Formulación de soluciones } \\
\text { o acciones }\end{array}$ & Toma de decisión & Implementación & Evaluación \\
\hline Actividades & Actividades & Actividades & Actividades & Actividades \\
\hline $\begin{array}{l}\text { Valores, acontecimientos, } \\
\text { intereses, demandas y } \\
\text { agenda pública }\end{array}$ & $\begin{array}{l}\text { Elaboración y valoración } \\
\text { de respuestas, selección } \\
\text { de criterios }\end{array}$ & $\begin{array}{l}\text { Encontrar una } \\
\text { coalición mayoritaria, } \\
\text { legitimación }\end{array}$ & $\begin{array}{l}\text { Ejecución, gestión, efectos } \\
\text { concretos }\end{array}$ & $\begin{array}{l}\text { Reacciones, juicio } \\
\text { sobre los efectos, } \\
\text { medición, valoración, } \\
\text { propuestas de reajuste }\end{array}$ \\
\hline Actores & Actores & Actores & Actores & Actores \\
\hline $\begin{array}{l}\text { Parlamentos, gremios, } \\
\text { Administración Pública, } \\
\text { organizaciones políticas y } \\
\text { sociales, etcétera }\end{array}$ & $\begin{array}{l}\text { Parlamentos, presidente, } \\
\text { ministro, gobernador, } \\
\text { alcalde, etcétera }\end{array}$ & $\begin{array}{l}\text { Administración } \\
\text { Pública, ONG, } \\
\text { empresa privada, } \\
\text { etcétera }\end{array}$ & $\begin{array}{l}\text { Medios, expertos, } \\
\text { Administración Pública, } \\
\text { responsables políticos, } \\
\text { organizaciones políiticas y } \\
\text { sociales, gremios, afectados, } \\
\text { ONG, etcétera }\end{array}$ & $\begin{array}{l}\text { Partidos, movimientos } \\
\text { sociales, gremios, } \\
\text { medios, políticos, } \\
\text { Administración Pública, } \\
\text { ONG, etcétera }\end{array}$ \\
\hline
\end{tabular}

Fuente: Tomado de Roth (2007, p. 69). 
pública involucra entre cuatro y cinco etapas, como las mencionadas anteriormente, y que se caracteriza en el ciclo de políticas públicas, que se puede sintetizar de la siguiente manera:

Compartel, entonces, se identifica como una política pública, puesto que esta emerge como un conjunto de acciones que representan las decisiones del Estado colombiano al intentar modificar las reglas de juego operantes hasta el año 1998 en cuanto a telefonía fija y acceso a Internet se refiere, por lo que la política de telecomunicaciones sociales Compartel aparece como la respuesta del Gobierno por intentar resolver la problemática de acceso universal que afrontaba Colombia hacia finales de la década de los noventa y que significaba garantizar que la ciudadanía tuviera acceso a servicios de telecomunicaciones; además, que se debía "llevar el acceso generalizado a los hogares de los servicios básicos de telecomunicaciones iniciando con el servicio de telefonía y posteriormente integrando otros servicios a medida que los avances tecnológicos y la disponibilidad de los recursos lo permitan", tal y como lo expresa el Decreto 899 de 1999. Es de destacar que en Colombia, en 1998, se presentaba una densidad, en líneas telefónicas por cada
100 habitantes, de 16,53 abonados en servicio y ocupaba el cuarto puesto en Latinoamérica (Hernández, 2010, p. 94).

En el análisis de las políticas públicas se dispone de diferentes modelos, enfoques y teorías; a efectos del análisis de la política de telecomunicaciones sociales Compartel se utilizó el enfoque de las teorías mixtas, ya que él permite tener una posición intermedia entre las teorías centradas en la sociedad y las teorías centradas en el Estado, además de que, de acuerdo con Roth (2006, p. 31), las teorías mixtas "permiten desarrollar investigaciones a partir de posiciones más abiertas", lo que redunda en la posibilidad de realizar aportes innovadores. En este sentido, el modelo de análisis usado es el Advocacy Coalitions de Paul Sabatier, el cual tiene en cuenta la información concerniente a los problemas que originan la política pública, la observación de estos problemas a largo plazo; el análisis de los subsistemas de política compuestos por actores públicos y privados, periodistas, investigadores y analistas de política, y los sistemas de creencias incorporados por los miembros de las coaliciones.

El modelo Advocacy Coalitions Framework (ACF) se sintetiza en la figura 1: 
Figura 1. Modelo Advocacy Coalitions Framework.

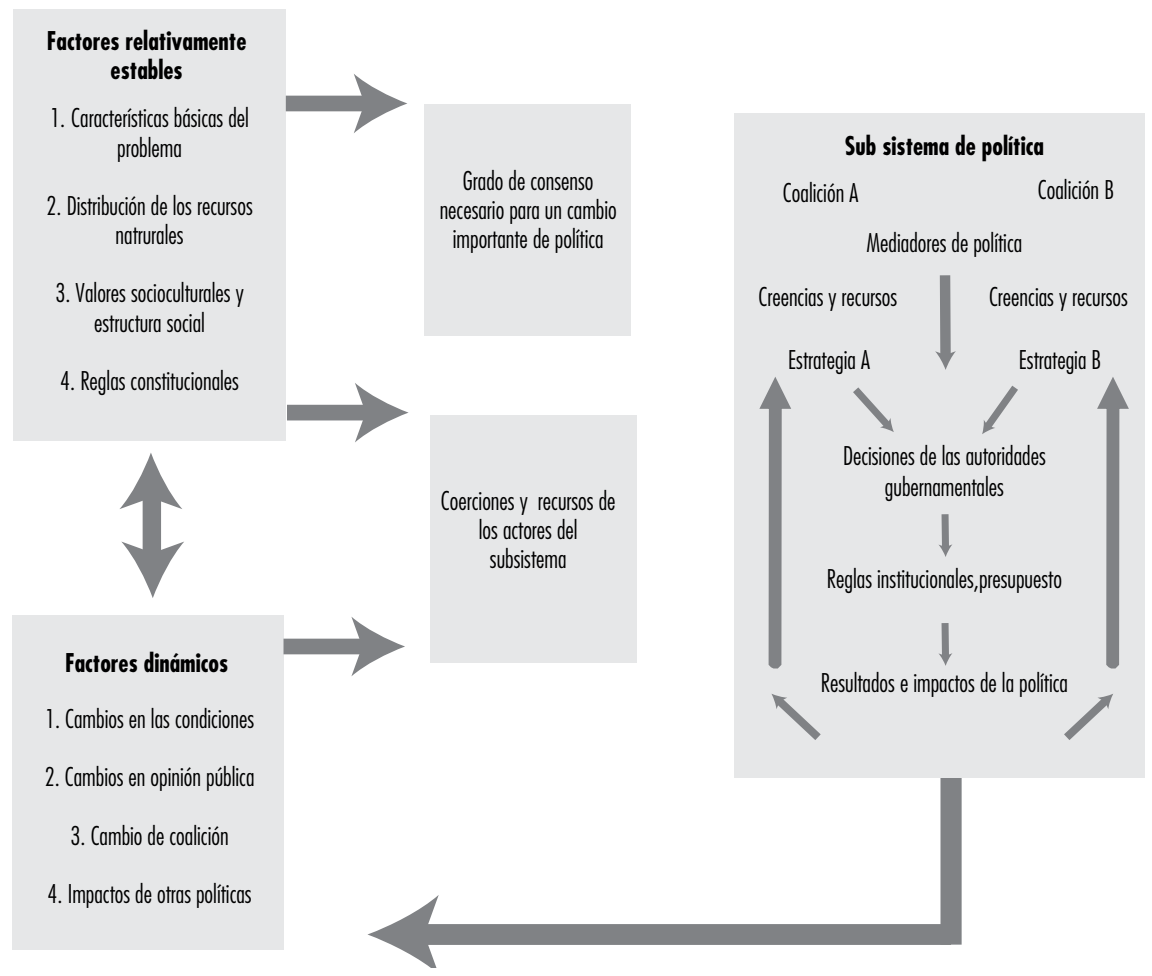

Fuente: Adaptación de Roth(2007).

Las coaliciones militantes (Advocacy Coalitions) son definidas por Sabatier (1988, p. 139, citado por Roth, 2007, p. 169) como:

Las personas pertenecientes a diferentes posiciones - responsables de entidades gubernamentales, congresistas, responsables políticos, responsables de gremios, investigadores- que comparten un sistema similar de creencias - serie de valores fundamentales, supuestos y percepciones de un problema específico- y que demuestran un cierto grado de coordinación en el tiempo.

Es, entonces, en el estudio y análisis de estas coaliciones, que se pueden identificar los cambios o la continuidad de las políticas públicas; en síntesis, estas coaliciones se caracterizan, $y$ se diferencian a la vez, por sus sistemas de creencias y sus intereses.

Roth (2007, p. 170) explica que los cambios que presentan las políticas 
públicas surgen como consecuencia de la lucha y el trabajo que realizan las coaliciones, las cuales buscan "hacer prevalecer su punto de vista, sus valores e intereses para lograr su dominio en la conducción de la política pública"; además, "en su trabajo de influencia sobre las políticas ellas intentan orientar la política hacia soluciones que correspondan a su sistema de intereses, valores y creencias" (Roth, 2007, p. 171); es en este aspecto donde el modelo ACF hace énfasis, ya que según Roth (2007, p. 172):

Es a partir de estos valores que la coalición que domina el subsistema va a percibir la problemática política y que va a imaginar, a construir y a implementar las medidas concretas (institucionales, legislación, reglamentos) y, por lo tanto, los cambios serán más o menos difíciles de realizar en función del grado de "profundidad" que implique el cambio para el sistema de valores de la coalición dominante.

Asimismo, expone Roth (2008, p. 3):

La posibilidad de introducir tanto elementos subjetivos, como las creencias y los valores, como elementos más objetivos relativos al contexto y a los intereses de los actores en un único esquema-guía, ofrece así la perspectiva de seguir a través del tiempo la influencia sobre la política pública de varios elementos: actores, contexto, ideas, información, cambios tecnológicos.

Al modelo ACF lo constituye un subsistema de política en el que se configuran las coaliciones, compuestas por instituciones, actores de la política tanto públicos como privados, funcionarios, pensadores, etc., los cuales intervienen en la problemática de la política pública; este subsistema se da en un contexto configurado por factores estables en el tiempo y por factores dinámicos o inestables. A los factores estables los integran las características del problema, entre las que se incluyen la descripción de la problemática en la que surge la política pública; la distribución de los recursos naturales, relacionada con las formas y maneras en que estos recursos influyen en la concepción de la política pública; los valores socioculturales y la estructura social, y las reglas constitucionales. Por su parte, a los factores dinámicos los componen los cambios en las condiciones socioeconómicas, es decir, las variables socioeconómicas que en el transcurso del tiempo son relativas a las condiciones del entorno y del mercado; la opinión pública, relacionada con elecciones o sondeos sobre temas 
prioritarios que puedan influir en los cambios de la política pública; el gobierno, especialmente relacionado con los cambios de los responsables de la política en las instituciones gubernamentales encargadas de la política pública y los impactos de otras políticas. "Ese entorno influye sobre el subsistema y sus actores, y actúa como un proveedor de coerciones, limitaciones y recursos", explica Roth (2008, p. 4).

Roth (2008, p. 4), entre los aspectos relevantes del modelo ACF, también resalta que este realiza una distinción entre cambios fundamentales y secundarios en la política:

Los primeros son asimilados a un cambio de comunidad de política dominante en el subsistema. Se asemeja, haciendo una analogía con la epistemología kuhniana, a un cambio de paradigma en la política. Estos cambios son relativamente escasos, ya que la mayoría de los cambios ocurren en el núcleo de la política (policy core) o, a un nivel aún más periférico, en los aspectos secundarios de la política (reglamentaciones y cambios institucionales que no cuestionen el núcleo o fundamento ni de la política (policy core) ni de las creencias (deep core) de la comunidad dominante acerca de la política en cuestión.

\section{Elementos metodológicos para el análisis de Compartel como política pública}

El enfoque de investigación con el cual se desarrolló este trabajo fue el cualitativo, en el que "se utiliza la recolección de datos sin medición numérica para describir o afinar preguntas de investigación en el proceso de interpretación" (Hernández, Fernández \& Baptista, 2008, p. 8); para ello se realizó la recolección de información en tres partes:

La primera, mediante una extensa indagación documental que contempló la revisión y análisis de textos de tipo académico (tesis de maestría y doctorado en la temática), revistas académicas, libros, artículos científicos, informes de investigación y memorias de eventos realizados sobre el tema a nivel nacional e internacional; se recolectaron documentos oficiales del Gobierno nacional, del Ministerio de Comunicación, el Departamento Nacional de Planeación, la Comisión de Regulación de Telecomunicaciones, el Congreso de la República, la Presidencia de la República, el Departamento Administrativo Nacional de Estadística (DANE) y los Conpes; además se identificaron informes y artículos comentados sobre los desarrollos de las TIC por parte 
de representantes de organizaciones sociales. La segunda parte contempló la identificación de actores clave testigos del proceso de desarrollo de la política de telecomunicaciones sociales Compartel que fueron consultados por medio de entrevistas semiestructuradas. Y la tercera parte se basó en la identificación de actores clave (fuentes primarias) que conocen el desarrollo de la política de telecomunicaciones sociales y cuyos testimonios fueron extraídos de otros trabajos documentales (libros, tesis, entrevistas) en los cuales fueron referenciados. También se consultaron testimonios, publicados en sitios web, de los actores clave (fuentes primarias) consultados directamente en las entrevistas semiestructuradas.

Cabe resaltar que las anteriores etapas no se desarrollaron de forma lineal, ya que, como lo aseguran Hernández et al. (2008, p. 582), “el proceso cualitativo no es lineal ni lleva una secuencia como el proceso cuantitativo. Las etapas constituyen más bien acciones que efectuamos para cumplir con los objetivos de la investigación y responder a las preguntas del estudio y se yuxtaponen".
De este modo la investigación cualitativa realizada se fundamentó en un proceso inductivo de explorar y describir para luego comprobar perspectivas teóricas que, en el caso de este trabajo, apunta a la descripción y explicación de los cambios en la política de telecomunicaciones sociales Compartel a partir del modelo de coaliciones militantes de Sabatier. Asimismo, para el caso del enfoque cualitativo:

La recolección de los datos consiste en obtener las perspectivas y puntos de vista de los participantes $[\ldots]$ el investigador pregunta cuestiones generales y abiertas, recaba datos expresados a través del lenguaje escrito, verbal y no verbal (Hernández et al., 2008, p. 8).

Como uno de los aspectos más importantes del modelo de coaliciones militantes está relacionado con el análisis de la política pública en un periodo no mayor de diez años, se realizó una línea de tiempo para identificar los eventos relevantes relacionados con los cambios que se presentaron en Colombia en materia de TIC en un contexto internacional, la cual se presenta a continuación: 
Tabla 2. Línea de tiempo 1998-2008.

\begin{tabular}{|c|c|c|c|c|}
\hline 1998 & 1999 & 2000 & 2002 & 2003 \\
\hline $\begin{array}{l}\text { Ausencia de articulación y } \\
\text { consolidación de políticas } \\
\text { públicas de TIC en } \\
\text { Colombia }\end{array}$ & $\begin{array}{l}\text { Plan Nacional de Desarrollo } \\
\text { "Cambio para construir } \\
\text { la paz" } \\
\text { Telefonía Social (Conpes } \\
\text { 3032) } \\
\\
\text { Computadores para Educar } \\
\text { (Conpes 3063) }\end{array}$ & $\begin{array}{l}\text { Agenda de } \\
\text { Conectividad } \\
\text { (Conpes 3072) }\end{array}$ & $\begin{array}{l}\text { Plan Nacional de Desarrollo } \\
\text { "Hacia un Estado } \\
\text { Comunitario" } \\
\\
\text { Lineamientos de política en } \\
\text { telecomunicaciones sociales } \\
\text { (Conpes 3171) }\end{array}$ & $\begin{array}{l}\text { Primera Cumbre } \\
\text { Mundial de la } \\
\text { Sociedad de la } \\
\text { Información }\end{array}$ \\
\hline
\end{tabular}

Fuente: Elaboración propia.

\begin{tabular}{|c|c|c|c|}
\hline 2005 & 2006 & 2007 & 2008 \\
\hline $\begin{array}{l}\text { Metas y estrategias de Colombia } \\
\text { para el logro de los Objetivos de } \\
\text { Desarrollo del Milenio (Conpes } \\
\text { Social 91) } \\
\text { Segunda Cumbre Mundial de la } \\
\text { Sociedad de la Información } \\
\text { Visión } 2019 \\
\text { Avance del Gobierno en Línea } \\
\text { (investig. Uniandes) }\end{array}$ & $\begin{array}{l}\text { Plan Nacional de Desarrollo } \\
\text { "Estado Comunitario: Desarrollo } \\
\text { para Todos" }\end{array}$ & $\begin{array}{l}\text { Lineamientos de Políitica } \\
\text { para reformular el } \\
\text { Programa Compartel de } \\
\text { Telecomunicaciones Sociales } \\
\text { (Conpes 3457) } \\
\text { Informe de Colombia sobre } \\
\text { los Objetivos de Desarrollo del } \\
\text { Milenio }\end{array}$ & $\begin{array}{l}\text { Agenda de Conectividad se } \\
\text { transforma en Gobierno en } \\
\text { Línea }\end{array}$ \\
\hline
\end{tabular}

Fuente: Elaboración propia. 


\section{Política de telecomunicaciones Compartel. Caracterización y análisis}

La investigación utiliza el modelo de análisis de política pública Advocacy Coalitions (coaliciones militantes) de Paul Sabatier, el cual tiene por objetivo poner de manifiesto los actores y los contextos de influencia que motivan los cambios en la política pública en el transcurso del tiempo. Este modelo de análisis es contemporáneo y ha sido poco utilizado en Latinoamérica para el análisis de las políticas públicas.

La política de telecomunicaciones sociales Compartel en Colombia se implementó con el documento Conpes 3032, que surge de las debilidades y deficiencias que se presentaron en la ejecución del Plan de Telefonía Social 1997-200 (Conpes 3032,1999 , p. 5) debido a que, como lo menciona dicho documento (pp. 4-5), "el plan era excesivamente ambicioso en las metas propuestas, pretendiendo atacar la problemática de las zonas urbanas y rurales simultáneamente con soluciones comunitarias y domiciliarias, sin establecer un orden de prioridades entre objetivos". En primera instancia, Compartel busca la promoción de la telefonía fija, especialmente en zonas rurales de Colombia, que para la época presentaba un nivel de densidad de líneas telefónicas relativamente bajo. Además, la idea que acompañaba este propósito era la de impulsar las telecomunicaciones para aumentar la competitividad en las regiones, idea que se sigue presentando en todo el tiempo de implementación de la política de Compartel y que se relaciona con la idea de que a mayor tecnología, más desarrollo económico y mayor productividad.

Meses después de promulgado el Conpes 3032 de 1999, se aprobó la ejecución de la Agenda de Conectividad mediante un nuevo Conpes, el 3072 de 2000, el cual contiene programas para la promoción de las TIC en diferentes sectores de la sociedad colombiana. En este Conpes el Programa Compartel de Internet Social aparece con la finalidad de promover los servicios de Internet en sectores de la población colombiana de bajos recursos económicos mediante la ampliación de la cobertura con la instalación de centros de acceso comunitario a Internet, patrocinados por el Gobierno, en las ciudades del país que no contaban con una infraestructura de telecomunicaciones suficiente y adecuada para prestar este servicio. 
Luego, el Conpes 3171 de 2002, que se denominó "Lineamientos en política sociales 2002-2003”, pretendió continuar y fortalecer algunos proyectos para lograr la conexión telefónica, la conectividad y el acceso a Internet en diversas zonas rurales de Colombia. En este documento Conpes se destaca que una de las preocupaciones del Gobierno se centra en la disminución de la brecha digital por el incremento de la cobertura y universalización de las telecomunicaciones; para ello se establecieron tres criterios importantes a seguir: 1) accesibilidad: significaba que el servicio telefónico estuviera disponible en el lugar y momento en que se requirieran; 2) la no discriminación, entendida como en el sentido de que todo usuario gozara de buen precio, servicio y calidad sin importar la localización geográfica; y 3) la asequibilidad, que define tarifas adecuadas para los usuarios (Hernández, 2011, p. 46).

En el 2007 la política de telecomunicaciones sociales Compartel se reformuló por medio del documento Conpes 3457, ya que el acceso a Internet y el uso de computadores en el 2005, de acuerdo con un diagnóstico realizado en el país, reflejó un rezago importante frente al uso de otras tecnologías como la comunicación por teléfonos celulares. En ese momento el panorama de acceso a las TIC se presentaba de la siguiente manera:

Tabla 3. Acceso a las TIC.

\begin{tabular}{|l|c|c|}
\hline \multicolumn{1}{|c|}{ Servicio } & $\begin{array}{c}\text { Colombia (Junio } \\
\text { 2006) }\end{array}$ & $\begin{array}{c}\text { Promedio } \\
\text { Latinoamérica } \\
\text { (2005) }\end{array}$ \\
\hline $\begin{array}{l}\text { Telefonía local } \\
\text { (líneas por 100 } \\
\text { habitantes) }\end{array}$ & 18,93 & 18,71 \\
\hline $\begin{array}{l}\text { Telefonía móvil } \\
\text { (terminales por } \\
\text { 100 habitantes) }\end{array}$ & 67,8 & 43,84 \\
\hline $\begin{array}{l}\text { Acceso a Internet } \\
\text { (usuarios por 100 } \\
\text { habitantes) }\end{array}$ & 13,2 & 12,93 \\
\hline $\begin{array}{l}\text { Acceso a Internet } \\
\text { de banda ancha } \\
\text { (usuarios por 100 } \\
\text { habitantes) }\end{array}$ & 6,1 & 14,4 \\
\hline $\begin{array}{l}\text { Acceso a PC } \\
\text { (PC por cada 100 } \\
\text { habitantes) }\end{array}$ & 5,4 & 9,5 \\
\hline
\end{tabular}

Fuentes: Unión Internacional de Telecomunicaciones (UIT), Comisión de regulación de Telecomunicaciones (CRT), cálculos Departamento Nacional de Planeación (DNP) (citado en documento Conpes 3457).

De acuerdo con lo anterior, los momentos de cambio de la política de telecomunicaciones sociales Compartel se caracterizan en los documentos Conpes mencionados. 


\section{Subsistema de política}

Uno de los elementos claves que explican los cambios de la política pública en el modelo ACF es el subsistema de política, ya que en este se identifican las coaliciones que actúan como protagonistas en los cambios de dicha política. En el proceso de cambio de la política de telecomunicaciones sociales Compartel se pueden identificar, a partir de la revisión documental realizada y de la contrastación con las fuentes primarias, básicamente dos coaliciones integradas por diversos actores participantes en las diferentes coaliciones políticas:

\section{Coalición uno: integracionista}

Se identifica de esta manera ya que se infiere a partir de la recolección de información de fuentes primarias y secundarias la intención de integrar el acceso universal, propiciado por la infraestructura en telecomunicaciones, que fue en principio el objetivo sustancial de la política de telecomunicaciones sociales Compartel, garantizar acceso universal con una estrategia de apropiación social para el uso de las TIC. La coalición uno, entonces, está integrada por dos sectores; por un lado la comunidad académica, y por otro, las organizaciones no gubernamentales.

\section{Comunidad académica}

Es reconocida como uno de los actores importantes en la coalición integracionista por ser motivadora de los cambios de política pública, de acuerdo con las fuentes primarias consultadas, ya que el Gobierno "sí está pensando las universidades, ya no solamente como un interlocutor para investigación, sino para construcción colectiva, como para ir haciendo el paso a paso y eso es importante" (J.M. Escobar, entrevista personal, 3 de septiembre, 2010); además, en temas de apropiación social de TIC "se ha logrado vincular a la academia... porque ese es el papel de la academia, pensar el país del futuro, el de entender el país del presente y entender también la historia" (C.A. Torres, entrevista personal, 10 de septiembre, 2010). La comunidad académica aparece como un interlocutor válido frente a procesos de uso y apropiación social de tecnologías como la informática e Internet, convirtiéndose en un actor protagónico en los procesos de política pública al respecto, en Colombia.

La comunidad académica está compuesta, en especial, por dos universidades que se han mostrado interesadas y activas en los desarrollos de la política de Compartel por 
medio de la integración a la Red $\mathrm{Na}$ cional de Telecentros, que ha sido el resultado de la unión de intereses comunes de las organizaciones que la componen: la Universidad Autónoma de Occidente y la Universidad del Cauca. Por su incidencia directa a través del proyecto piloto "Gestión del conocimiento en telecentros Compartel”, se caracteriza la Universidad Autónoma de Occidente como miembro importante y representativo de esta comunidad.

- Grupo de Investigación en Comunicación para el Desarrollo, Universidad Autónoma de Occidente (Cali, Colombia). Categorizado en C por el Departamento Administrativo de Ciencia, Tecnología e Innovación (Colciencias), tiene como propósito generar propuestas teórico-metodológicas que orienten y hagan viables procesos de incorporación estratégica de las TIC en el desarrollo comunitario por medio de dos líneas de investigación: los Sistemas integrales de información y comunicación para la generación de espacios conjuntos de reflexión temática enfocados en el fortalecimiento social y cultural desde la dimensión de una comunicación dialógica orientada en el uso y apropiación de las TIC, y el Modelo de telecentros, que trabaja, entre otros elementos, las estrategias de sostenibilidad implementadas por organizaciones locales (Universidad Autónoma de Occidente). Este grupo de investigación trabaja desde el año 2002 proyectos de uso y apropiación social de TIC, específicamente en el suroccidente colombiano; aporta al conocimiento, utilización y apropiación de herramientas informáticas y de Internet en comunidades rurales y urbanas marginadas mediante proyectos de telecentros.

El papel de la academia como parte de una coalición del subsistema de la política de telecomunicaciones sociales Compartel representa para Jorge Mauricio Escobar, director de este grupo de investigación:

Dos escenarios, uno donde la academia ha aportado en investigación con estudios serios, econométricos, insumos con fuentes fidedignas, $y$ otro escenario donde la apuesta es el proceso, el apalancamiento de procesos a través de la propuesta y ejecución de proyectos piloto (J.M. Escobar, entrevista personal, 3 de septiembre, 2010). 


\section{Organizaciones no gubernamentales}

Como parte de la coalición integracionista se encuentran las organizaciones no gubernamentales. En el desarrollo del trabajo de investigación se reconocieron cuatro organizaciones no gubernamentales que representan a la sociedad civil como movilizadoras e impulsadoras de cambios en la política de telecomunicaciones sociales Compartel, tal y como lo expresa César Torres, director de Apropiación Social del Ministerio de Tecnologías de la Información y las Comunicaciones:

Por el lado de apropiación hemos logrado vincular organizaciones no gubernamentales; tenemos a Colnodo, a Makaia, hemos ido creciendo en la vinculación de ONG, a que asuman roles [sic] reales de convocatoria, de capacitación, de diseño de propuestas y de consecución de apoyos.

Y de acuerdo con Jorge Mauricio Escobar, director del Grupo de Investigación en Comunicación para el Desarrollo:

El trabajo que han hecho las ONG en el tema de apropiación, haciendo ver la importancia de desarrollar trabajos colegiados y en alianza para tener ejercicios de aprendizaje, ha sido bueno, ahí tienen nombre propio Colnodo, Makaia y Corporación Colombia Digital" (J.M. Escobar, entrevista personal, 3 de septiembre, 2010).

Las organizaciones no gubernamentales representativas de esta coalición son:

- Colnodo. Está situada en Bogotá. Fue fundada en 1994 y es una asociación de siete organizaciones no gubernamentales sin fines de lucro. Sus ejes de trabajo son programas informáticos libres, género y desarrollo sostenible. Entre sus actividades estratégicas relacionadas con las TIC se encuentran: 1) gobierno en línea y democracia electrónica; 2) promoción de la participación ciudadana en el uso y apropiación de las TIC; 3) políticas de tecnologías de información y comunicación; 4) investigación, desarrollo y gestión del conocimiento en TIC.

El trabajo de Colnodo en la apropiación social de las TIC es reconocido por César Torres:

Gracias a que el aliado, que es Colnodo, que a su turno nos trajo y nos aproximó a unos nuevos y 
muy importantes aliados como la Universidad Autónoma de Occidente, como Makaia, es como los telecentros se convirtieron en unos escenarios reales del proceso de apropiación y el concepto de sostenibilidad ha venido dando un giro hacia cómo las comunidades pueden hacer de las TIC algo que mejore la calidad de vida (C. Torres, entrevista web, julio, 2010).

Es de destacar que Colnodo ha venido trabajando, desde la aparición de Internet en Colombia, en sistemas de comunicación electrónica, con el objetivo de intercambiar información, en primera instancia entre organizaciones no gubernamentales, espectro que en la actualidad se ha ampliado.

- Corporación Colombia Digital. Su centro de acción es la ciudad de Bogotá. Fue fundada en el 2002 por varias instituciones de los sectores público, privado $\mathrm{y}$ académico. Es una organización sin ánimo de lucro, de tipo privado, que busca promover el uso y apropiación de las TIC en diferentes sectores de la vida social y económica del país. Entre sus servicios se encuentran: elaboración, ejecución y administración de proyectos en torno a las TIC; capacitaciones y procesos de formación en TIC y asesorías para la estructuración de proyectos TIC. Su sitio web: www. colombiadigital.net, contiene la compilación de una gran cantidad de materiales e información de TIC de Colombia y el mundo, es un centro de consulta en línea en el que se encuentran proyectos, políticas e iniciativas de TIC de diversa índole.

- Makaia. Geográficamente está ubicada en la ciudad de Medellín, segunda ciudad en importancia de Colombia. Makaia significa en miskito (lengua indígena de Honduras), "construir" o "hacer". El nombre representa el objetivo de Makaia de establecer alianzas y relaciones orientadas al desarrollo social y económico. Esta organización promueve el fortalecimiento institucional de organizaciones de la sociedad civil, gobiernos locales, entidades sin ánimo de lucro e iniciativas de responsabilidad social -empresarial del sector de lucro comprometidas con el desarrollo social y económico. Sus servicios se centran en dos áreas temáticas: 1) consultorías para la internacionalización; 2) asesorías especializadas en TIC cuya experiencia en este ámbito 
está respaldada por la gerencia de proyectos en el sector público y no gubernamental en Colombia, en organismos multilaterales y la banca internacional de desarrollo, entre los que se encuentran "Acompañamiento técnico y social a organizaciones sociales y comunitarias de base para el montaje de telecentros comunitarios", "Apropiación social de telecentros Compartel", "Empresarismo social para telecentros - Fases 1 y 2", y el "V Encuentro Nacional de Telecentros", por citar algunos.

Entre los aportes significativos que ha generado Makaia en el tema de la apropiación social de las TIC por medio de los telecentros se encuentra el modelo de empresariado social para fomentar la autosostenibilidad de estos, que consiste en abordar, de forma paralela: 1) la conectividad y el acceso; 2) su apropiación; 3) contenidos y herramientas con enfoque en la conectividad (Botero et al., 2009, p. 7).

- Red Nacional de Telecentros. La Red Nacional de Telecentros es una iniciativa de organizaciones académicas, no gubernamentales y del Estado colombiano, que decidieron unir sus esfuerzos para trabajar en torno al desarrollo y fortalecimiento de los telecentros en Colombia y de las comunidades que los integran; asimismo, tiene como visión ser generadora de nuevas políticas públicas y proyectos para el desarrollo. La red es dirigida por un comité coordinador, conformado por el Ministerio de Tecnologías de la Información y las Comunicaciones, Colnodo, Corporación Makaia, Universidad Autónoma de Occidente (Grupo de Investigación en Comunicación para el Desarrollo), Empresa de Telecomunicaciones de Bogotá, Corporación Colombia Digital, Universidad del Cauca y la Fundación Telecentre.org, como miembro honorario. Entre los ejes estratégicos de trabajo de la red se encuentran: 1) el fortalecimiento de redes mediante la creación de alianzas que promuevan acciones para la incidencia en políticas públicas y la organización de encuentros regionales; 2 ) la gestión del conocimiento como eje estratégico para impulsar nuevos productos del conocimiento y espacios para el intercambio de saberes entre diversos actores 
de la red; 3) las redes regionales como puntos focales para coordinar el trabajo en las regiones (departamentos, municipios, zonas) e interactuar con la red nacional; 4) información y comunicación para fortalecer los canales de interacción con los miembros de la red; 5) formación y capacitación a través de talleres y encuentros y por medio de la Academia Nacional de Telecentros; 6) investigación e incidencia en políticas de TIC, cuyo objetivo es realizar investigaciones sobre temas TIC para formular recomendaciones y proponer acciones de incidencia en el tema (Paz, 2009, pp. 7-8).

En ese sentido Olga Paz (entrevista personal, 10 de septiembre, 2010) opina que entre las organizaciones que han incidido para los cambios en la política de telecomunicaciones sociales Compartel se encuentra la Red Nacional de Telecentros; además, como lo afirma Jorge Mauricio Escobar:

Estamos teniendo alta incidencia en política pública porque como red tenemos unos propósitos, una filosofía, un plan de desarrollo; la incidencia ha sido macro inicialmente por medio de proyectos piloto $y$ luego vinimos a nivel regional y somos consultados y tenidos en cuenta por parte del ministerio, por eso considero que hemos avanzado mucho (J.M. Escobar, entrevista personal, 3 de septiembre, 2010).

\section{Sistema de creencias de la coalición integracionista}

La coalición integracionista se identifica, entre sus diversos actores, por un sistema de creencias fundamentado en: 1) el interés de trabajar alrededor de la apropiación de las TIC como un factor fundamental que contribuye al desarrollo de las comunidades; 2) la convicción de trabajar en beneficio de las comunidades con la utilización de las TIC para generar impacto social; 3) generar conocimiento y desarrollar procesos de apropiación social de las TIC para fortalecer social, económica y políticamente a las comunidades, lo que se puede resumir en que "este uso de las TIC contribuye a la mejora de la calidad de vida" (Botero et al., 2009, p. 1).

\section{Coalición dos: normativa}

La segunda coalición en el subsistema de la política de telecomunicaciones sociales Compartel se denomina normativa, pues las características que predominan en esta coalición son el 
alcanzar las metas y los estándares de penetración de las telecomunicaciones en Colombia propiciadas en su mayor parte por los organismos internacionales que buscan promover el uso de las TIC en las sociedades con el fin de consolidar la sociedad de la información y del conocimiento. Esta coalición está compuesta por el Gobierno, representado por el Ministerio de Tecnologías de la Información y las Comunicaciones, y por organismos y movimientos internacionales como la Unión Internacional de Telecomunicaciones (UIT), la Cumbre de la Sociedad de la Información y los Objetivos de Desarrollo del Milenio. A continuación se caracterizan estos actores integrantes de la coalición normativa:

\section{Ministerio de Tecnologías de la Información y las Comunicaciones}

Este ministerio, de acuerdo con la Ley 1341 de 2009, tiene entre sus funciones: 1) diseñar, adoptar y promover las políticas, planes, programas y proyectos del sector de las TIC; 2) definir, adoptar y promover las políticas, planes y programas tendientes a incrementar y facilitar el acceso de todos los habitantes del territorio nacional a las TIC y a sus beneficios, para lo cual debe diseñar, formular y proponer políticas, planes y programas que garanticen el acceso y la implantación de las TIC con el propósito de fomentar su uso como soporte del crecimiento y aumento de la competitividad del país en los distintos sectores y el mejoramiento de la calidad de vida de la comunidad; el acceso a mercados del sector productivo y el acceso equitativo a oportunidades de educación, trabajo, salud, justicia, cultura y recreación; apoyar al Estado en el acceso y uso de las TIC para facilitar y optimizar la gestión de los organismos gubernamentales y la contratación administrativa transparente $y$ eficiente; prestar mejores servicios a los ciudadanos, apoyar la formulación de los lineamientos generales de difusión de la información que generen los organismos del Gobierno y efectuar las recomendaciones que considere indicadas para lograr que esta sea en forma ágil y oportuna, e igualmente, diseñar y desarrollar estrategias masivas que expliquen a los ciudadanos las utilidades y potencialidades de las TIC; 3) promover el establecimiento de una cultura de las TIC en el país, por medio de programas y proyectos que favorezcan la apropiación y masificación de las tecnologías, como instrumentos que facilitan el bienestar y el desarrollo personal y social; 4) ejercer la representación internacional de 
Colombia en el campo de las TIC, especialmente ante los organismos internacionales del sector; 5) ejecutar los tratados y convenios sobre TIC ratificados por el país en temas relacionados con el espectro radioeléctrico y los servicios postales; 6) evaluar la penetración, uso y comportamiento de las TIC en el entorno socioeconómico nacional, así como su incidencia en los planes y programas que implemente o apoye; 7) propender por la utilización de las TIC para mejorar la competitividad del país.

Es así como, de acuerdo con Luis Fernando Lozano, coordinador de Operaciones de Compartel:

El Estado es el que siempre ha sido el mayor jalonador de la política; en un principio la política inicia solamente con la ejecución de recursos estatales, antes todo era centralizado, todo lo hacía el Ministerio de Comunicaciones en su época, hoy Ministerio de TIC; aquí se formulaban, se planeaban, se contrataban y se ejecutaban los proyectos, hoy estamos dando un giro completamente en este tema porque ya en las regiones se han apropiado del tema de las comunicaciones sociales, por ejemplo, ya se ven gobernaciones que apropian recursos para desarrollar proyectos, entonces, no solo somos nosotros los que vamos e imponemos, sino que entre todos estamos aportando (L.F. Lozano, entrevista personal, 4 de octubre, 2010).

\section{Organismos internacionales}

Unión Internacional de Telecomunicaciones (UIT). Es un organismo de la Organización de las Naciones Unidas (ONU) conformado por 192 naciones partes y más de 700 miembros del sector de las telecomunicaciones en el mundo. La UIT congrega, entonces, gobierno y sector privado, y coordina tres sectores fundamentales de las telecomunicaciones: radiocomunicaciones, normalización y desarrollo. Fue, además, el principal organismo patrocinador de la Cumbre Mundial sobre la Sociedad de la Información. Tiene su sede en Ginebra y su misión es la de:

Permitir el crecimiento y el desarrollo sostenible de las redes de telecomunicaciones y de información, y facilitar el acceso universal para que todos en todas partes puedan participar en la economía y la sociedad mundial de la información y beneficiarse de ellas (UIT, 2010).

Para la UIT la posibilidad de comunicar libremente es condición sine qua non de un mundo más equitativo, próspero y pacífico, por lo cual la UIT ayuda a movilizar los recursos técnicos, financieros y humanos necesarios para concretizar este propósito. Colombia es miembro de la UIT desde el 25 de agosto de 1914. 
La UIT es gestora de dos de los cuatro índices comúnmente aceptados a nivel mundial para medir el estado de desarrollo y apropiación de las TIC en los países, uno de estos índices es el Reporte Mundial de Gobierno Electrónico (Global e-Government Readiness Report):

A través del índice se relacionan las TIC con los temas de productividad y competitividad de los países como preparación para el gobierno electrónico y [la] participación electrónica. De esta manera, se estima el uso de Internet por parte de los Estados para la provisión de información, productos y servicios a la población, mediante una evaluación de la infraestructura en telecomunicaciones, los sitios web del Estado y la capacidad del capital humano para usarlos. Adicionalmente, el reporte examina los programas de gobierno para incentivar la participación de los ciudadanos en las políticas públicas en tres aspectos: tipo de información disponible en línea, los mecanismos de consulta y la existencia de herramientas para la toma de decisiones en línea a partir de los insumos de los ciudadanos (DNP, 2007, pp. 37-38).

Otro de los índices de medición para el desarrollo de las TIC, generado a partir de la UIT, es el Índice de Oportunidad Digital (IOD, por sus siglas en inglés):
Diseñado para hacer seguimiento al progreso de un país en el cierre de la brecha digital, tanto interna como comparativamente con otros países, y la implementación de los compromisos de la Cumbre Mundial de la Sociedad de la Información. Para tales efectos, el IOD se basa en tres dimensiones, las cuales reúnen varios indicadores a su vez: la oportunidad, la infraestructura y la utilización (DNP, 2007, p. 37).

- Cumbre Mundial de la Sociedad de la Información. Se desarrolló en dos fases: la primera en Ginebra, en diciembre del 2003, y la segunda en Túnez, en noviembre del 2005. Representantes de los Gobiernos de 175 y 174 países respectivamente, asistieron a la primera y a la segunda cumbre.

Los compromisos adquiridos por parte de los Gobiernos en la primera cumbre fueron: 1) colaborar para ampliar el acceso a la infraestructura y las TIC, así como a la información y al conocimiento; fomentar la capacidad; reforzar la confianza y la seguridad en la utilización de las TIC; crear un entorno propicio a todos los niveles; desarrollar y ampliar las aplicaciones TIC; 2) el reconocimiento de la conectividad como un factor indispensable en la creación de la sociedad de la información. El acceso 
universal, ubicuo, equitativo y asequible a la infraestructura y los servicios TIC constituye uno de los retos de la sociedad de la información y debe ser un objetivo de todas las partes interesadas que participan en su creación; 3) garantizar una infraestructura de red y aplicaciones de las TIC que estén bien desarrolladas, adaptadas a las condiciones regionales, nacionales y locales, fácilmente accesibles y asequibles y que, de ser posible, utilicen en mayor medida la banda ancha y otras tecnologías innovadoras, ya que estas pueden acelerar el progreso económico y social de los países, así como el bienestar de todas las personas, las comunidades y los pueblos; 4) Internet se ha convertido en un recurso global disponible para el público, y su gestión debe ser una de las cuestiones esenciales del programa de la sociedad de la información. La gestión internacional de Internet debe ser multilateral, transparente y democrática, y contar con la plena participación de los Gobiernos, el sector privado, la sociedad civil y las organizaciones internacionales.

En el 2005, en la segunda cumbre, los Gobiernos se comprometieron, entre otras cosas, a: 1) evaluar y seguir de cerca los progresos tendientes al cierre de la brecha digital, en que se tuvieran en cuenta los diferentes niveles de desarrollo, con miras a lograr las metas y objetivos de desarrollo internacionalmente acordados, incluidos los Objetivos de Desarrollo del Milenio, y a evaluar la eficacia de la inversión y los esfuerzos de cooperación internacional encaminados a la construcción de la sociedad de la información; 2) instar a los Gobiernos a que, con el uso del potencial de las TIC, se crearan sistemas públicos de información sobre leyes y reglamentos, considerar un desarrollo mayor de los puntos de acceso públicos y apoyar una amplia disponibilidad de esta información; 3) promover el acceso universal, ubicuo, equitativo y asequible a las TIC, incluidos el diseño universal y las tecnologías auxiliares para todos, con atención especial a los discapacitados, con el objetivo de garantizar una distribución más uniforme de sus beneficios entre las sociedades y dentro de cada una de ellas, reducir la brecha digital a fin de crear oportunidades digitales para todos y beneficiarse del potencial que brindan las TIC para el desarrollo; 4) tomar las medidas necesarias para garantizar que todos los países del mundo dispongan de un acceso equitativo y asequible a las TIC para que sus beneficios en los campos del desarrollo socioeconómico y del 
cierre de la brecha digital sean verdaderamente integradores (Compromiso de Túnez, 2005).

- Objetivos de Desarrollo del Milenio. En la Asamblea General de la ONU del año 2000, las 189 naciones participantes se comprometieron a adoptar los estándares fijados en ella para la erradicación de la pobreza por medio de ocho objetivos que deben ser cumplidos para el 2015 . El objetivo 8 declara "fomentar una asociación mundial para el desarrollo", en la cual se reconoce que el acceso y uso de las TIC constituye una eficaz herramienta para la inserción económica y cultural, ya que aumenta la probabilidad de avanzar en la consecución de los Objetivos de Desarrollo del Milenio al ser un instrumento útil en materia de conocimientos para los propósitos de la lucha contra la pobreza, las enfermedades, el hambre y el analfabetismo, entre otros. Para medir los indicadores de cumplimiento se fija, por cada cien habitantes, el número de líneas de teléfono, el de abonados a teléfonos celulares y el de usuarios a Internet.

\section{Sistema de creencias de la coalición normativa}

Lo que prevalece en esta coalición es el compromiso del Gobierno colombiano por cumplir con los indicadores de TIC, en razón de lo pactado ante instancias internacionales que buscan la promoción y la inclusión de las TIC en las sociedades al propender por el desarrollo y el mejoramiento de la calidad de vida entre sus usuarios, como lo menciona Luis Fernando Lozano:

\begin{abstract}
Colombia asiste a foros y eventos internacionales en los cuales se firman pactos en pro del desarrollo y metas que se tienen que alcanzar $y$ acorde con esos compromisos internacionales que se adquieren la política pública se enfoca a cumplir con esas metas (L.F. Lozano, entrevista personal, 4 de octubre, 2010).
\end{abstract}

En el modelo ACF uno de los elementos a destacar en las relaciones entre las coaliciones es el sistema de creencias que las une o las diferencia. En el caso de la política de telecomunicaciones sociales de Compartel, el subsistema de esta política, en el que se configuran las coaliciones, refleja varios aspectos que diferencian y unen a las coaliciones a la vez, integrándolas, al final, en torno al mismo sistema de 
creencias. Estos aspectos se determinaron en el análisis de los datos documentales recolectados y los datos cualitativos resultado de las entrevistas a las fuentes primarias.

\section{Momentos de cambio en la política de telecomunicaciones sociales Compartel}

Como resultado de la implementación del Conpes 3032 de 1999, en Colombia se instalaron puntos Compartel de telecomunicaciones comunitarias en 7415 localidades rurales de todo el territorio nacional, "garantizando la prestación de servicios básicos de telecomunicaciones a casi cuatro millones de habitantes de las zonas rurales más remotas del territorio nacional, a tarifas asequibles y con niveles de calidad adecuados" (Conpes 3171, 2002, p. 6). En el 2002 los puntos Compartel estaban instalados en el territorio nacional así:

Durante este periodo también se instalaron puntos Compartel con acceso a Internet y se instalaron redes conmutadas de acceso a la red en gran parte de la geografía nacional. En un primer momento se expresó, entonces, cómo la política de telecomunicaciones sociales tuvo sus orígenes en la necesidad de suplir con cobertura telefónica a zonas apartadas de Colombia, la cual rápidamente se transformó en la ampliación de esa cobertura telefónica a otros servicios de telecomunicaciones, como el acceso a Internet (Hernández, 2011, p. 100).

\section{Figura 2. Localidades con puntos} Compartel en junio de 2002

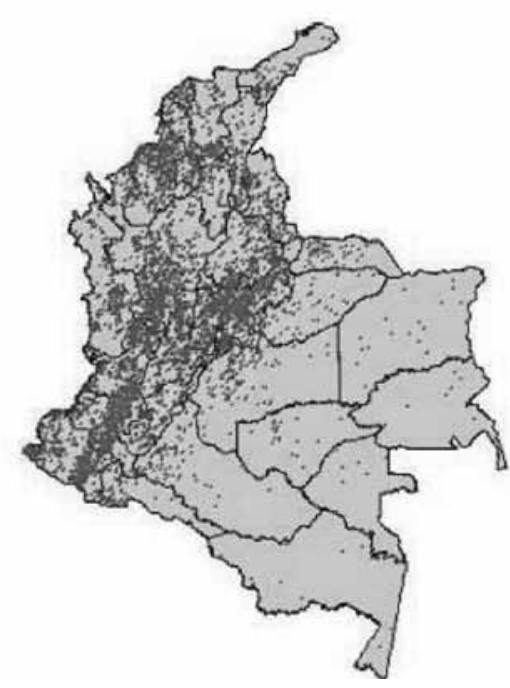

Fuente: Programa Compartel

En el 2002 se identificó la existencia de una gran demanda del servicio telefónico en áreas rurales alejadas, donde sus habitantes deben recorrer grandes distancias para acceder al servicio telefónico, aunque se destaca que 940 cabeceras municipales del país, es decir, el 86\% del total, cuentan con centros de acceso comunitario a Internet cuyas tarifas para la prestación de los servicios 
se consideran acordes con la capacidad de pago de los usuarios (Conpes 3172, 2002, p. 12). Además, con el ánimo de incrementar la cobertura a Internet aparecieron, en ese momento, los telecentros o centros de acceso comunitario a la red como una estrategia para fortalecer, ampliar y garantizar el acceso a Internet en regiones del país en las que hasta el momento ese acceso no era posible. Esta estrategia se denominó Fase III de Internet Social e instaló 500 nuevos telecentros en las cabeceras municipales del país donde no se prestaba el servicio.

Las fases I, II y III de Internet Social presentan el siguiente impacto:

Tabla 4. Fases I, II y III de Internet Social.

\begin{tabular}{|c|c|c|c|}
\hline & Fase I & Fase II & Fase III \\
\hline $\begin{array}{l}\text { Cabeceras } \\
\text { municipales }\end{array}$ & 636 & 261 & 244 \\
\hline $\begin{array}{l}\text { Centros } \\
\text { poblados }\end{array}$ & 34 & & 297 \\
\hline $\begin{array}{l}\text { Guarniciones } \\
\text { militares }\end{array}$ & & & 9 \\
\hline $\begin{array}{l}\text { Población } \\
\text { beneficiada }\end{array}$ & $\begin{array}{l}2500000 \\
\text { hab. aprox. }\end{array}$ & $\begin{array}{l}900000 \\
\text { hab. } \\
\text { aprox. }\end{array}$ & $\begin{array}{l}850000 \\
\text { hab. } \\
\text { Aprox. }\end{array}$ \\
\hline $\begin{array}{l}\text { Total } \\
\text { telecentros }\end{array}$ & 670 & 270 & 500 \\
\hline
\end{tabular}

Fuente: Programa Compartel. Elaboración propia.

En síntesis, la población beneficiada con las fases I, II y III de Internet
Social desarrollado por la política de telecomunicaciones sociales Compartel representó hasta el 2002 un impacto en más de cuatro millones de colombianos beneficiados con la cobertura y el acceso a Internet, lo que representa el diez por ciento de la población colombiana.

Nuevos lineamientos para la política de Compartel se presentan en relación con su reestructuración. Jorge Mauricio Escobar considera que esos nuevos lineamientos son el resultado de los cambios en la concepción por parte del Ministerio de las Tecnologías de la Información y las Comunicaciones en cuanto a que:

El ministerio ha tenido una apertura de orden conceptual interesante, ha logrado entender el problema del uso de las tecnologías en función, no solamente del primer paradigma de modernización que fue todo lo de infraestructura, reducción de la brecha digital a través de equipamiento y grandes inversiones, sino de la mirada de un componente de apropiación social de tecnología (J. M. Escobar, entrevista personal, 3 de septiembre, 2010).

A partir del documento Conpes 3457, "Lineamientos para reformular el Programa Compartel de Telecomunicaciones Sociales", del 29 de enero de 2007, se trazó un nuevo 
direccionamiento en la política de telecomunicaciones sociales que corresponde a garantizar, por parte del Estado, el acceso a las TIC, pero también propende por el aprovechamiento de estas tecnologías por parte de los ciudadanos, pues como lo menciona María del Rosario Guerra (entrevista personal, 14 de abril, 2010), "existe una barrera cultural en el uso de las TIC, mayoritariamente en los adultos, mientras a los jóvenes no se necesita impulsarlos mucho, lamentablemente a adulto sí". El diagnóstico presentado en el Conpes 3457 (2007, p. 9) evidencia que en los años anteriores al 2007:

Los programas diseñados hasta el momento (Telefonía rural comunitaria, Telecentros, Conectividad a Internet de banda ancha para instituciones públicas y Planes bianuales de ampliación y reposición de redes-Compartel) han tenido un énfasis en el mejoramiento del acceso a las telecomunicaciones desde el punto de vista de la infraestructura, con algunos esfuerzos en la sensibilización de la población beneficiada para la incorporación de las TIC en sus actividades.

Asimismo, se resalta que pese al mejoramiento de la cobertura y la prestación de servicios de telecomunicaciones, el país para esta época continuaba por debajo del promedio latinoamericano en indicadores como acceso a Internet, banda ancha y masificación y uso de las TIC, a lo que se le suma la existencia de una brecha interna con respecto a las condiciones de acceso y aprovechamiento a estas tecnologías (Conpes 3457, 2007, p. 16). En ese sentido, Olga Paz (entrevista personal, 10 de septiembre, 2010) comenta:

Compartel se creó con un énfasis de acceso público a telefonía rural y a Internet, ese fue el enfoque, y si se evalúa desde ahí hasta el año 2006 podemos decir que se cumplió con el objetivo, pero para muchas organizaciones sociales no estaba cumpliendo un objetivo de apropiación de TIC y eso en nuestra opinión era relevante.

El documento Conpes 3457 expone, entonces, que para la ejecución de los lineamientos de política se debe tener en cuenta:

[...] como objetivo, consolidar las condiciones de acceso a las TIC y promover la apropiación de dichas tecnologías por parte de la población de menores ingresos del país; igualmente, buscar potenciar las oportunidades que ofrecen las TIC para mejorar la competitividad de las regiones de menor desarrollo y de las mipymes (Conpes 3457, 2007, p. 21).

Respecto a esto, para Olga Paz (coordinadora de Proyectos de Colnodo) el Conpes 3457 es producto 
de los resultados obtenidos en el proyecto "Gestión del conocimiento e intercambio de experiencias entre telecentros Compartel y telecentros comunitarios" coordinado por Colnodo e implementado por la Universidad Autónoma de Occidente, pues:

Compartel se dio cuenta [de] que efectivamente con procesos de apropiación, los telecentros podían cambiar, se podían generar impactos, por lo cual, posterior al proyecto se formula el Conpes, y nosotros nos adjudicamos ese logro porque seguramente sin ese proyecto el Conpes no hubiera existido... A partir de allí, la política de Compartel tiene el componente de apropiación, que antes no tenía (citado en Hernández, 2011, p. 114).

En síntesis, y de acuerdo con el modelo Advocacy Coalitions Framework y uno de sus pilares relacionado con los problemas en torno a la política, la política de telecomunicaciones sociales Compartel se caracteriza a partir de la necesidad que se presenta en la nación colombiana del acceso universal a las telecomunicaciones, en primera instancia, y en segunda instancia, la de procurar por parte del Gobierno el servicio universal de telecomunicaciones a los ciudadanos, necesidad que presenta transformaciones en el tiempo cuando aparecen nuevas necesidades en el ámbito de las telecomunicaciones tales como el acceso, específico a Internet y, por último, la apropiación social de las TIC por parte de las comunidades que tienen el acceso y el servicio en sus localidades.

\section{Conclusiones}

Colombia, durante los últimos años, ha tenido una evolución en los índices de TIC, ha logrado ir cumpliendo con los compromisos adquiridos a nivel internacional sobre el desarrollo de las TIC en el país, y la implementación de la política de telecomunicaciones sociales ha desempeñado un papel determinante en la consecución de estas metas ya que sus programas y proyectos han motivado la penetración, el acceso, el uso y la apropiación de las TIC en gran parte del territorio nacional y los subsistemas de política representan un factor determinante para ello, pues:

Los cambios que presentan las políticas públicas surgen como consecuencia de la lucha y el trabajo que realizan las coaliciones, las cuales buscan hacer [sic] su punto de vista, sus valores e intereses para lograr su dominio de conducción de la política pública, además, en su trabajo de influencia sobre las políticas ellas intentan orientar la política 
hacia soluciones que correspondan a su sistema de intereses, valores y creencias (Roth, 2007, pp. 170-171).

El Gobierno colombiano cambió su concepción instrumental y técnica sobre las TIC, especialmente Internet, y evolucionó hacia una mirada más amplia del uso de estas tecnologías, como lo asegura Luis Fernando Lozano:

Nosotros pasamos de ser un proyecto ciento por ciento fundamentalmente de infraestructura, donde lo único que hacíamos era ir y tender redes, a un concepto mucho más general que tiene que ser la apropiación, donde las TIC sean un instrumento que ayude a mejorar la calidad de vida, y esto por sí solo la tecnología no lo hace, tiene que haber un componente de apropiación (L.F. Lozano, entrevista personal, 4 de octubre, 2010).

La incidencia en la política de telecomunicaciones sociales Compartel de organizaciones como Colnodo ha representado un reto para estas en términos de gestión del conocimiento, ya que la integración con la política les ha exigido la sistematización de sus experiencias y la elaboración de productos de conocimiento como libros, cartillas y desarrollo de metodologías; tal y como lo expresa Olga Paz: "Nosotros no teníamos metodologías escritas, todo lo teníamos en la cabeza, pero no teníamos nada escrito, no teníamos materiales, en el marco de los proyectos no habíamos gestionado el conocimiento, entonces empezamos desde ahí, a convertir las experiencias en productos para compartir conocimiento". De igual manera, la academia ha desempeñado un papel vital en la incidencia de políticas públicas de TIC en Colombia, ya que a partir de sus adelantos en investigación la articulación academia-comunidad-Gobierno ha contribuido al debate y a la construcción de líneas de acción que motivan actividades conducentes a la generación de conocimientos que, para el caso de la política de telecomunicaciones sociales Compartel, ha representado metodologías y sistematización de experiencias que han redundado en el mejoramiento y perfeccionamiento de la política Compartel para el cumplimiento de sus objetivos. Se refleja que la sinergia entre academia y organizaciones no gubernamentales, en asocio con el Gobierno, potencializa iniciativas y proyectos, logra objetivos comunes y evita duplicar esfuerzos.

Por último, el compromiso de Colombia ante los entes internacionales de garantizar el acceso a la 
información a través de Internet a los colombianos y la inserción a la sociedad del conocimiento de los ciudadanos presionó al Gobierno a adelantar una política de telecomunicaciones sociales que asegurará cobertura y servicios de acceso universal, lo que apalanca, a su vez, otras políticas gubernamentales con el ánimo de cumplir las metas e indicadores de acceso y uso de las TIC, fijadas por organismos internacionales como la Unión Internacional de Telecomunicaciones. De esta manera, el desarrollo de la política de telecomunicaciones sociales Compartel se encuadra en la agenda pública internacional sobre la democratización de la información y el acceso, uso y apropiación de las TIC para el desarrollo.

\section{Referencias}

Diccionario de la lengua española. Recuperado el 20 de agosto de 2011, en www.rae.es.

Compartel (2004). Programa Compartel de Telecomunicaciones Sociales. Estudio de caso. Recuperado el 4 de abril de 2010, en http://www.itu. int/ITU-D/fg7/case_library/case_study_2/Americas/Columbia.pdf.
Colombia, Congreso Nacional de la República (2009, 30 de julio). Ley 1341 del 30 de julio de 2009, por la cual se definen principios y conceptos sobre la sociedad de la información y la organización de las tecnologías de la información y las comunicaciones (TIC), se crea la Agencia Nacional de Espectro y se dictan otras disposiciones. Diario Oficial, 47 426, Bogotá.

Colombia, Ministerio de Defensa Nacional (1999, 24 de mayo), Decreto número 899 del 24 de mayo de 1999, por medio del cual se fijan las políticas para el fomento de los programas de telefonía social y se reglamentan las funciones del Fondo de Comunicaciones en la materia. Diario Oficial, 43 588, Bogotá.

Colombia, Consejo Nacional de Política Económica y Social (Conpes), documento 3032 de 1999.

3072 de 2000.

3171 de 2002.

3457 de 2007.

Colombia, Departamento Nacional de Planeación (1999). La telefonía en Colombia, 1998. Documento sectorial. 
Colnodo. Recuperado el 2 de octubre de 2010, en http:// www.colnodo. apc.org,

Compartel. Recuperado el 2 de octubre de 2010, en http://www.compartel.gov.co.

Escobar, J.M. (2010, 3 de septiembre). Entrevistado por Hernández, M., Cali-Valle.

Hernández, M. (2011). Análisis de la política de telecomunicaciones sociales Compartel dentro del marco de las políticas públicas en tecnologías de la información y las comunicaciones Internet- en Colombia, 1998-2009.

Hernández, R., Fernández-Collado, C. \& Baptista, P. (2008). Metodología de la investigación. México: McGraw-Hill.

Lozano, L. (2010, 4 de octubre). Entrevistado por Hernández, M., Bogotá.

Makaia. Recuperado el 27 de septiembre de 2010, en http://www. makaia.org.

Nelson, B.J. (2001). Políticas públicas y administración: una visión general. En R.E. Godin y H. Klingemann (Edits.). Nuevo manual en ciencia política (Vol. 2, pp. 795-847). España: Istmo.
Paz, O. (2010, 10 de septiembre). Entrevistada por Hernández, M., Bogotá. Roth, A. (2006). Políticas públicas. Formulación, implementación y evaluación. Bogotá: Aurora.

(2007). Teorías y marcos de análisis para las políticas públicas. En A. Vargas et al. Fundamentos de políticas públicas. Bogotá: Universidad Nacional de Colombia. (2007). Enfoques y teorías para el análisis de las políticas públicas, cambio de la acción pública y transformaciones del Estado. En J.F. Jolly y J.I. Cuervo (Edits.). Lecturas de políticas públicas. Bogotá: Universidad Externado de Colombia.

Salazar, C. (1999). Las políticas públicas: marco de apoyo a lo comunicacional. Lo político desde la comunicación, 1, 50.

Sandoval, C. (2009, enero-junio). Gobiernos electrónicos y acción colectiva a través de Internet: dinámicas en la región andina. Revista de Relaciones Internacionales, Estrategia y Seguridad, 4 (1), 31-53.

Torres, C.A. (2010, julio). Entrevistado por Cruz, C., en http://www. 
telecentros.org.co/index.shtml?apc $=\& \mathrm{~s}=\mathrm{k} \& \mathrm{x}=22415$. (2010, 10 de septiembre). Entrevistado por Hernández, M., Bogotá. UIT, Unión Internacional de Telecomunicaciones. Recuperado de 2010, en http://www.itu.int.
Universidad Autónoma de Occidente. Recuperado el 3 de octubre de 2010, en http://www.uao.edu.co.

Vargas, A. (1999). El Estado y las políticas públicas. Bogotá: Almudena. 